Häringer, P. and Borrmann A. (2019). "Improving flow in Concrete Constructions by Avoiding Spatial Conflicts Between Pour Cycles." In: Proc. 27th Annual Conference of the International. Group for Lean Construction (IGLC), Pasquire C. and Hamzeh F.R. (ed.), Dublin, Ireland, pp. 323-334 DOI: https://doi.org/10.24928/2019/0189. Available at: 〈www.iglc.net>.

\title{
IMPROVING FLOW IN CONCRETE CONSTRUCTIONS BY AVOIDING SPATIAL CONFLICTS BETWEEN POUR CYCLES
}

\author{
Paul Häringer ${ }^{1}$ und André Borrmann²
}

\begin{abstract}
Supervisors and formwork engineers divide construction sections into multiple pour cycles in order to achieve a good production flow in concrete constructions. A pour cycle consists of one or more disconnected casting segments. Casting segments in the successor pour cycle often fill the gaps between the disconnected casting segments in the predecessor pour cycle. We call such a meeting of two neighbour casting segments "topological dependency" because it effects spatial conflicts between pour cycles and different trades cannot work on the next casting segment until the previous ones are completely finished. Because of the long curing times, trade crews have to wait or move to other locations. In this research, we introduce a new structure of a cycle planning option, which can avoid such spatial conflicts. We evaluated our cycle planning option by using a stochastic discrete event simulation model and compared it with three practical cycle planning options from one supervisor and two formwork engineers. The criteria for the evaluation were the total construction time as well as the stable production rate and balanced work. In addition, we discuss the potential benefit by using a mix of concrete precast elements and casting segments to achieve an even better production flow.
\end{abstract}

\section{KEYWORDS}

Work flow, flow integration, simulation, building information modelling (BIM)

\section{INTRODUCTION}

Supervisors and formwork engineers use cycle planning (CP) to plan cast in-situ constructions. They divide a construction section such as a floor into multiple pour cycles in order to achieve a good production flow in concrete constructions. A pour cycle consists of one or more disconnected casting segments. Current analysis of practical CP options show that there is a fundamental problem regarding the achievement of a good production flow due to dependencies and time variations.

\footnotetext{
${ }^{1}$ Research Associate, Chair of Computational Modeling and Simulation, TUM Department for Civil, Geo and Environmental Engineering, Technical University of Munich, Germany, paul.haeringer@tum.de

${ }^{2}$ Professor, Chair of Computational Modeling and Simulation, TUM Department for Civil, Geo and Environmental Engineering, Technical University of Munich, Germany, andre.borrmann@tum.de
} 
Gregory A. Howell (1999) mentioned the importance of dependencies and variations when he postulated that the combined effect of dependency and variation in managing the interaction between activities is essential if we are to complete projects in the shortest possible time. Dependencies in terms of space are very important because, unlike manufacturing where the work moves to people, on a construction site the people move to the work (Ballard and Howell 1998). The work is performed at locations on site so that space becomes an important resource, which has to be considered when planning construction projects (Frandson et al. 2015). Flow represents a fundamental concept in the production design process, it incorporates both continuity (absence of stoppages) and speed (Ballard et al. 2001).

Technological reasons, especially the shrinking process of cast in-situ concrete constructions, are leading to the construction of smaller, disconnected casting segments for a pour cycle. The time needed for the activity of pouring the concrete as well as the subsequent curing time greatly depends on external conditions. Unfavourable weather and temperature conditions in particular lengthen the time needed for pouring and extend the duration of curing. Therefore, the time between pouring and striking the formwork may vary greatly between different pour cycles. Consequently, if casting segments in the successor pour cycle meet or touch any other casting segments in the predecessor pour cycle (defined as a topological dependency), unpredicted waiting times can occur.

This research shows the impact of topological dependencies, which have the capacity to slow down and even stop the production process. It outlines an alternative concept for creating and structuring a CP option in terms of avoiding topological dependencies.

\section{BACKGROUND}

In an earlier case study, we investigated how supervisors and formwork engineers were planning to construct cast in-situ constructions for a residential building (Häringer and Borrmann 2018). The task at hand to create a suitable plan for the construction of the concrete walls and to maintain a working time frame of ten working days. The unit under construction consisted of four apartments and a staircase with an elevator shaft. We received three different $\mathrm{CP}$ options made by three different practitioners. The $\mathrm{CP}$ options are called CP-A, CP-B and CP-C.

In a $\mathrm{CP}$ option, one single casting segment is defined as zone and a group of segments as area, representing a pour cycle. The term zone is used as a construction segment which is fully covered by formwork elements (Biruk and Jaskowski 2017). Both the terms zone and area are used in the Lean Construction literature when referring to takt time planning (TTP) (Frandson et al. 2013).

Independent of the number of trade crews, a zone with dependencies to a zone in the predecessor area can only begin when the zone in the successor area is completely finished. Formwork elements block the space needed to construct the neighbouring casting segment in the predecessor area. Figure 1 illustrates this space conflict for CP-B. Zones 4, 5, 6, and 7 have topological dependencies to their neighbouring zones in the successor area. For example, Zone 5 meets Zones 2 and 3 - the bulkhead formwork blocks the space for Zone 5, 
so that a trade crew cannot fully set the formwork for Zone 5 and has to wait until the construction process of Zones 2 and 3 is finished.
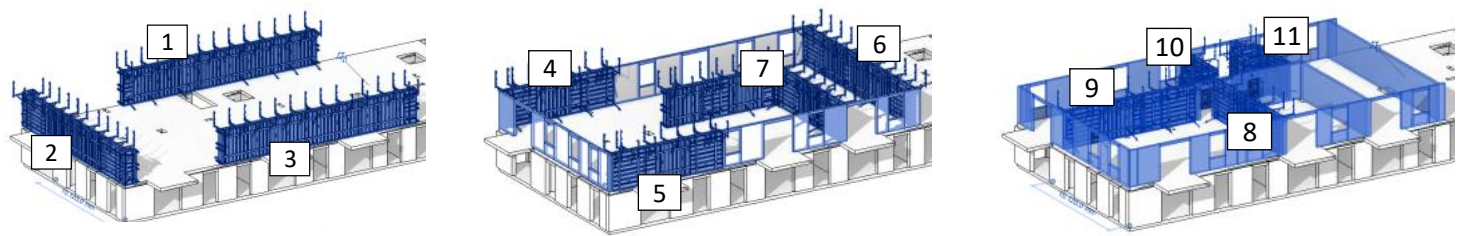

Figure 1: A typical structure of a $\mathrm{CP}$ - the walls of a construction unit (like a floor) are divided into segments (the zones numbered from 1 to 7 ), a group of disconnected segments represents the areas, area 1 with Zones 1 to 3 , Area 2 with Zones 4 to 7 and Area 4 with Zones 8 to 11

\section{RESEARCH METHOD}

The research method is based on three steps. In the first step, we created a basic generic building information model, which can represent all the different $\mathrm{CP}$ options. In the second step, we used this model to create the three different practitioner CP options, as well as to generate a partly automated optimised CP option, which has fewer topological dependencies between pour cycles. In the third and last step, we implemented a stochastic discrete event simulation model to analyse and evaluate the generated $\mathrm{CP}$ options in relation to the total construction time, stable production rate and balanced work. According to Sacks (2016), the stable production rate is the variation within each trade crew's takt time and balanced work is the variation between different trade crews.

The process of constructing a casting segment is a sequential execution of the following six activities:

[1] Setting the formwork on one side (setting 1); [2] Mounting the rebar (reinforcing); [3] Setting the formwork on the second side (setting 2), [4] Pouring the concrete (pouring);

[5] Waiting until the concrete is strong enough (curing) and [6] Removing the formwork (striking).

Taking into account the fact that the supervisor as well as the both formwork engineers used slightly different values for the performance factors, we decided to use a triangle distribution and set a minimum, mean and maximum value for each activity's performance factor (PF). A triangle distribution is linear and values close to the mean value are more likely to be chosen. This type of distribution has been successfully applied in Monte Carlo simulations to estimate the probability of the total time of reinforced concrete work (Hofstadler 2007).

The supervisor mentioned a time of around 12 hours as a practical value for the curing time. This means that the concrete can be poured in the evening so the formwork panels can start being removed the next day. The formwork engineers told us that it could take up to 36 hours. We decided to set the curing time to 24 hours, a time, which lies between 12 and 36 hours. 
Table 1 shows the minimum, mean and maximum values employed of the performance factors for each activity.

Table 1: Minimum, mean and maximum values of the performance factors (PFs) for each activity as input for the triangle distribution

\begin{tabular}{lllll}
\hline Type of PF & Min Value & Mean Value & Max Value & Unit \\
\hline PF_setting1 & 0.13 & 0.15 & 0.17 & $\mathrm{~h} / \mathrm{m}^{2}$ \\
PF_setting2 & 0.13 & 0.15 & 0.17 & $\mathrm{~h} / \mathrm{m}^{2}$ \\
PF_reinforcing & 14 & 16 & 18 & $\mathrm{~h} / \mathrm{tonnes}$ \\
PF_pouring & 0.5 & 0.7 & 0.9 & $\mathrm{~h} / \mathrm{m}^{3}$ \\
PF_curing & 24 & 24 & 24 & $\mathrm{~h}$ \\
PF_striking & 0.11 & 0.13 & 0.15 & $\mathrm{~h} / \mathrm{m}^{2}$ \\
\hline
\end{tabular}

The supervisor as well as the both formwork engineers calculated on using two trade crews; trade crew 1 executed the activities setting1, setting2, pouring and striking, while trade crew 2 performed the activity of reinforcing. Taking into account the fact that they used slightly different numbers of workers in a trade crew, we decided to use an empirical distribution. Table 2 details the different number of workers employed in each trade crew and their frequency as a percentage in order to depict the probability for the empirical distribution.

Table 2: Number of workers for trade crew 1 and 2

\begin{tabular}{|c|c|c|c|}
\hline $\begin{array}{l}\text { Number of workers in } \\
\text { trade crew } 1\end{array}$ & Frequency [\%] & $\begin{array}{l}\text { Number of workers in } \\
\text { trade crew } 2\end{array}$ & Frequency [\%] \\
\hline 2 & 30 & 3 & 30 \\
\hline 3 & 40 & 4 & 40 \\
\hline 4 & 30 & 5 & 30 \\
\hline
\end{tabular}

The equation for the duration of an activity is:

$$
\begin{gathered}
\text { Duration acitivity }=\frac{\text { Amount of } x \text { for a zone }(\text { casting segment }) * P F_{y}}{\text { number of workers in a trade crew } z} ; \\
x \hat{=} \text { unit of measurement }\left[\mathrm{m}^{2}, \mathrm{~m}^{3}, \text { tonnes }\right] ; y \hat{=} \text { type of } P F ; z \hat{=} \text { trade crew for the activity }
\end{gathered}
$$

\section{CYCLE PLANNING DESIGN PROCESS}

The cycle planning design process describes our workflow for generating, analysing and evaluating different CP options. A location breakdown structure (LBS) is used to define the location for a CP option. Kenley and Seppänen (2010) describe three different hierarchy levels for LBSs. The highest hierarchy level divides buildings into sections and risers, the middle hierarchy into floors and the lowest into apartments. We propose a middle hierarchy 
level such as the floor represented in Figure 1 in order to begin detailed construction planning.

\section{Model And Data Preparation}

The design of the building represented by a building information model (BIM) describes the value that has to be produced for the customer. In order to be flexible as to how to construct the walls, we split them into small slices. This splitting follows a rule of not splitting through openings and it is performed automatically by using a splitting algorithm, which we devised ourselves, for Autodesk Revit (Häringer and Borrmann 2018).

Pursuing the concept of achieving a good location flow without any waiting times between the pour cycles, we needed to reduce the topological dependencies. Consequently, we propose a method where neighbouring zones are separated from each other in order to reduce the dependencies between zones in successor areas. According to Borrmann and Rank (2009), the solid representation of a BIM is suitable for obtaining topological information and thus for deriving the spatial relationships between elements. Consequently, we used graph theory to get the topological relations between every element in a BIM. In our case, an adjacency matrix was suitable for representing the relations between all the elements. If two elements are neighbours and the second element has the same alignment (for example it still runs along the $\mathrm{x}$-axis in the coordination system), then it is given the value "AA" in the adjacency matrix. If the second element does not have the same alignment (for instance the second element is rotated through $90^{\circ}$ and now runs along the $\mathrm{y}$-axis), then it represents a cross member and the value becomes "AT". The first element in such an "AT" relation represents a node with high topological dependency (NWHTD).

Figure 2 illustrates the process of splitting the model into small slices (split model) and in Figure 3 the creation of a CP option (CP-B) from the split model can be seen. Figure 4 represents our optimised $\mathrm{CP}$ option by using NWHTDs to decouple the zones between them.

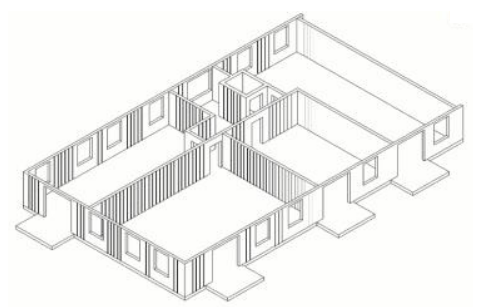

Figure 2: Splitting process

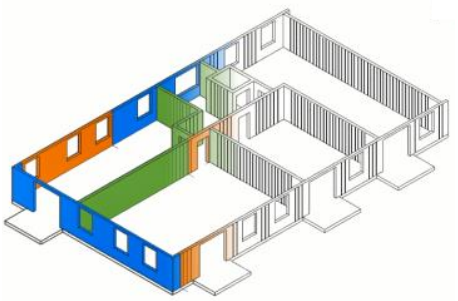

Figure 3: Creation of a CP option

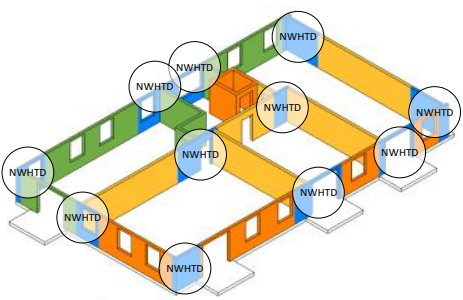

Figure 4: optimised CP option

\section{Planning and Checking}

The "Planning and Checking" describes the procedure for creating or generating a CP option. After identifying all the NWHTDs, we calculated the distance between them using a breadth-first search algorithm (Cormen 2005). All the elements (the small slices) between two NWHTDs are our zones. We used the information on the distance measured to adjust 
the zones, so that the length of every zone became similar. The idea is that similar zones could be constructed in the same way just as with standardised products.

An algorithm takes two NWHTDs and checks whether the length between them is between $7.00 \mathrm{~m}$ and $9.00 \mathrm{~m}$. If it is more than $9.00 \mathrm{~m}$, slices are then added to the NWHTDs until the length is under $8.50 \mathrm{~m}$; if it is less, then nothing need be done. This algorithm works well if the distance between all the NWHTDs is similar. However, how should one proceed with small distances that are around $1.00 \mathrm{~m}$ in length? They are too small to have a zone of their own. One method could be to add them to those which are in the range of approx. 4, 5 or $6 \mathrm{~m}$. Thus, a smaller zone can approach $8 \mathrm{~m}$ in length. No algorithm has yet been implemented for this, so we calculated it manually to check whether it could be a good way to handle this problem or not.

The Last Planner System (LPS) identifies which work should and could be done, then tracks the commitments for what will be done (Ballard and Howell 2003). It focuses more on the social process of planning and commitments to improve quality and reliability by involving the trade crews (Seppänen et al. 2010). The process "Planning and Checking" needs human decisions, especially to determine the number of areas and to allocate the zones to areas. This is a crucial process because it has an immediate impact on the trade crew's work. Referring to the LPS, this process should provide information and possible suggestions on how the work can be done.

We received the information from the supervisor that external walls needed to be constructed first. The main reason was to avoid blocking the walking routes with interior walls. Another reason was that it provided more safety because the exterior walls prevent falls from a height. The experts proposed solutions with three or four areas (pour cycles). The estimate of the number of areas was based on a mix of expert knowledge, referring to the total amount of concrete needed, the total wall length and the curing time for each pour cycle. Figure 5 and Figure 6 illustrate our proposed optimised CP option (CP-D). The numbers in Figure 5 and Figure 6 represent the zones and the same colour defines the pour cycles (areas). In order to compare CP-D with the other three $\mathrm{CP}$ options, we considered a group of NWHTDs as one zone. We defined three zones, numbered as 1, 2, and 3, as shown in Figure 5.

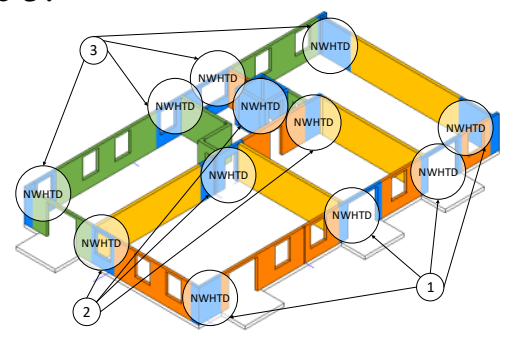

Figure 5: Optimised CP option with Zones 1 to 3

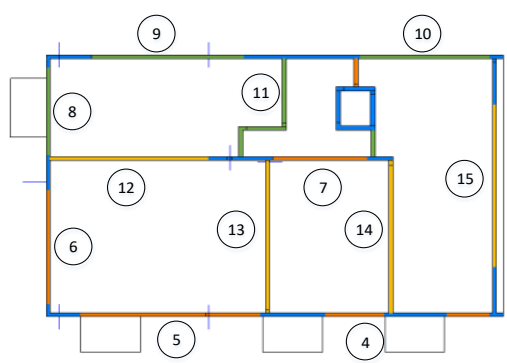

Figure 6: Optimised CP option with Zones 4 to 16.

The NWHTDs are dynamic and change their size, so that we can obtain almost similar zones. Apart from zone 4, which consists of two smaller segments, all the zones are between $5.5 \mathrm{~m}$ and a maximum of $8.5 \mathrm{~m}$. 


\section{Simulation AND EVALUATION}

The "Simulation and Evaluation" describes the procedure for simulating and evaluating a $\mathrm{CP}$ option. The process of constructing a casting segment (zone) of a $\mathrm{CP}$ option, as well as the input parameters and variables used are described in the research method section.

The sequential execution of pour cycles (areas), as well as the casting segments (zones) of area 1, are fixed in our simulation model and both are determined by the user before the simulation starts. For example, CP-B ( Figure 1) starts with Area 1, which includes the fixed sequence of Zone 1, Zone 2 and Zone 3 followed by Area 2 and Area 3. The sequence of the zones in Areas 2 and 3 is dependent on whether there is a connection to the last zone in the predecessor area or not. It should be remembered that the information on the connection, as well as the type of connection, is defined in the adjacency matrix. If the last zone of an area is in the curing activity, then trade crews will be available to work on the next zone in the successor area. However, the trade crew can only work on the next zone if there is no connection, otherwise there will be a spatial conflict. The model prevents such a spatial conflict by changing and sorting the sequence of zones in the successor areas, so that "not connected" zones will be executed first. However, this is only possible if the CP option has one or more "not connected" zones between the predecessor and successor area.

On construction sites, there is frequently no fixed shift schedule. The work usually starts at 7 am and ends at any time between $5 \mathrm{pm}$ and $9 \mathrm{pm}$. The shift end often depends on whether the trade crew wants or has to pour a casting segment that day or not. A limited shift schedule could give a disadvantage or advantage to a particular CP option, so we decided to simulate it with a shift schedule of 24 hours and 7 days.

We wanted to evaluate and compare the different $\mathrm{CP}$ options in terms of the total construction time (TCT), balanced work (BW) and stable production rate (SPR) criteria. First, we simulated three scenarios, each for one of these criteria. Before we started with a fourth scenario, which had the goal of finding the best $\mathrm{CP}$ option by considering all the criteria in an evaluation function, we had to choose the weight of each criterion and determine its impact factors. The following equation represents the evaluation function by considering all the above-mentioned criteria.

$$
\text { eval }(C P \text { option })=T C T * \text { factorTCT }+B W * \text { factor } B W+S P R * \text { factor } S P R
$$

The factor weights the criteria and is zero if the criteria are not considered. If only one criterion is considered, the value of the factor for this criterion is one. The simulation results of the first three scenarios showed that the TCT value of the eval (CP option) evaluation function was roughly 55 times higher than the SPR value and 350 times higher than the $\mathrm{BW}$ value. It is clear that the TCT value is very high because it takes the total construction time into consideration, which is in the range of approx. four to six days. The SPR value was measured as the mean deviation within a trade crew for each activity over all locations (zones). The BW value was measured as the mean deviation between two trade crews and their activities. Both values were in the range of minutes and hours but the BW value was approximately seven times lower than the SPR value. One reason could be that the BW value was only measured between the activities setting1 and reinforcing as well between reinforcing and setting2. These are the activities that are performed by different trade crews. 
Consequently, the factor values for the fourth scenario, called composite criteria (CC), was one for the TCT factor, 55 for the SPR factor and 350 for the BW factor. We performed 3000 simulation runs per scenario to find the best candidate in each $\mathrm{CP}$ option. The best candidates in each CP option and scenario are listed in Table 3. The CP option, which had the best candidate of all $\mathrm{CP}$ options, is shown underlined and in italics. The results show that the best candidates for scenario [1] TCT and [3] SPR, as well as for [2] BW and [3] $\mathrm{CC}$ were the same.

Table 3: Simulation results for each scenario [1] TCT, [2] BW, [3] SPR and [4] CC with the values of the most suitable candidates for each $\mathrm{CP}$ option

\begin{tabular}{|c|c|c|c|c|c|c|c|c|c|c|c|c|}
\hline & $\begin{array}{l}0 \\
\frac{0}{0} \\
\bar{t} \\
\text { 응 } \\
0\end{array}$ & 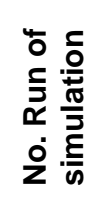 & 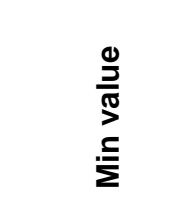 & 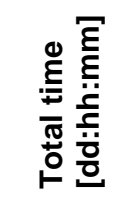 & $\begin{array}{l}\bar{z} \\
\text { do } \\
\overline{0} \\
\dot{0} \\
\dot{z}\end{array}$ & 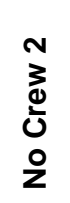 & 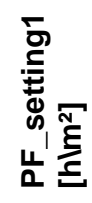 & 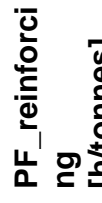 & 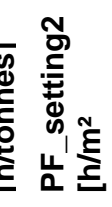 & 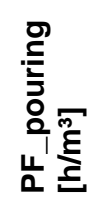 & 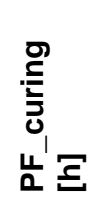 & 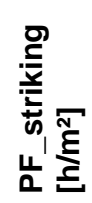 \\
\hline \multirow{6}{*}{ 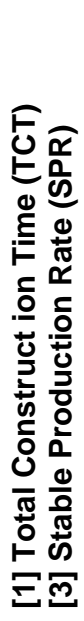 } & $\begin{array}{l}\text { CP- } \\
\text { A }\end{array}$ & 881 & $\begin{array}{l}520302 \text { [1] } \\
15013 \text { [3] }\end{array}$ & $6: 00: 31$ & 4 & 5 & 0.14 & 16.88 & 0.14 & 0.56 & 24.00 & 0.13 \\
\hline & & & $400446[1]$ & & & & & & & & & \\
\hline & $\begin{array}{l}\text { CP- } \\
\text { B }\end{array}$ & 2194 & 7169 [2] & $4: 15: 14$ & 4 & 5 & 0.14 & 15.66 & 0.14 & 0.56 & 24.00 & 0.13 \\
\hline & $\frac{C P-}{C}$ & 881 & 492734 [1] & $5 \cdot 16 \cdot 52$ & 4 & 5 & 0.14 & 16.88 & 0.14 & 0.56 & 24.00 & 0.13 \\
\hline & C & & $\underline{3874[3]}$ & & & & & & & & & \\
\hline & $\frac{C P}{\underline{D}}$ & $\underline{881}$ & $\frac{350605[1]}{5120[3]}$ & $\underline{4: 01: 23}$ & 4 & $\underline{5}$ & $\underline{0.14}$ & $\underline{16.88}$ & 0.14 & $\underline{0.56}$ & $\underline{24.00}$ & 0.13 \\
\hline \multirow{4}{*}{ 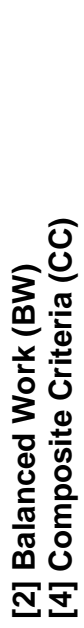 } & $\begin{array}{l}\text { CP- } \\
\text { A }\end{array}$ & 1055 & $\begin{array}{l}1487 \text { [2] } \\
2129504 \text { [4] }\end{array}$ & $6: 15: 58$ & 3 & 5 & 0.14 & 14.93 & 0.14 & 0.62 & 24.00 & 0.13 \\
\hline & $\begin{array}{l}\text { CP- } \\
\text { B }\end{array}$ & 112 & $\begin{array}{l}1184 \text { [2] } \\
1388235 \text { [4] }\end{array}$ & 5:08:01 & 3 & 5 & 0.15 & 14.98 & 0.15 & 0.61 & 24.00 & 0.14 \\
\hline & $\begin{array}{l}\text { CP- } \\
\mathrm{C}\end{array}$ & 2517 & $\begin{array}{l}1108 \text { [2] } \\
1218661[4]\end{array}$ & $6: 10: 41$ & 3 & 5 & 0.15 & 14.98 & 0.15 & 0.81 & 24.00 & 0.13 \\
\hline & $\frac{C P-}{\underline{D}}$ & $\underline{2596}$ & 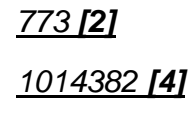 & $4: 11: 23$ & $\underline{3}$ & $\underline{5}$ & $\underline{0.16}$ & $\underline{15.06}$ & $\underline{0.16}$ & $\underline{0.69}$ & $\underline{24.00}$ & $\underline{0.12}$ \\
\hline
\end{tabular}


The optimised CP option (CP-D) provided the best candidate for scenario [1] Total Construction Time, [2] Balanced work and [4] Composite Criteria. The simulation run No. 2596 represents the best candidate for CP-D in scenario [2] as well in [4]. Apart from the results in scenario [3] SPR, the optimised CP option (CP-D) provided the best candidate.

A standard line-of-balance chart can illustrate the location and trade flow in one diagram (Sacks, 2016). Consequently, it is suitable for illustrating and analysing our simulation results. The structures of $\mathrm{CP}-\mathrm{A}, \mathrm{CP}-\mathrm{B}$ and $\mathrm{CP}-\mathrm{C}$ are similar. As a result, all of them have many topological dependencies between pour cycles (areas). Both CP-B and our generated and optimised CP-D with fewer topological dependencies are displayed in this paper, so it is reasonable to show the effect of topological dependencies by comparing CP-B with CP-D. Because of the topological dependencies, the trade crews in CB-B are unable to work during the curing activity (Figure 7). The trade crews in CP-D can work during the curing activity after all NWHTDs in Area 1 have been completely constructed (Figure 8). Consequently, the trade crews are not forced to pour the castings segments at the end of the day in order to use the time over night for the curing. CP-D can prevent waiting times and improve flow, however this might give rise to more inventory (formwork panels).

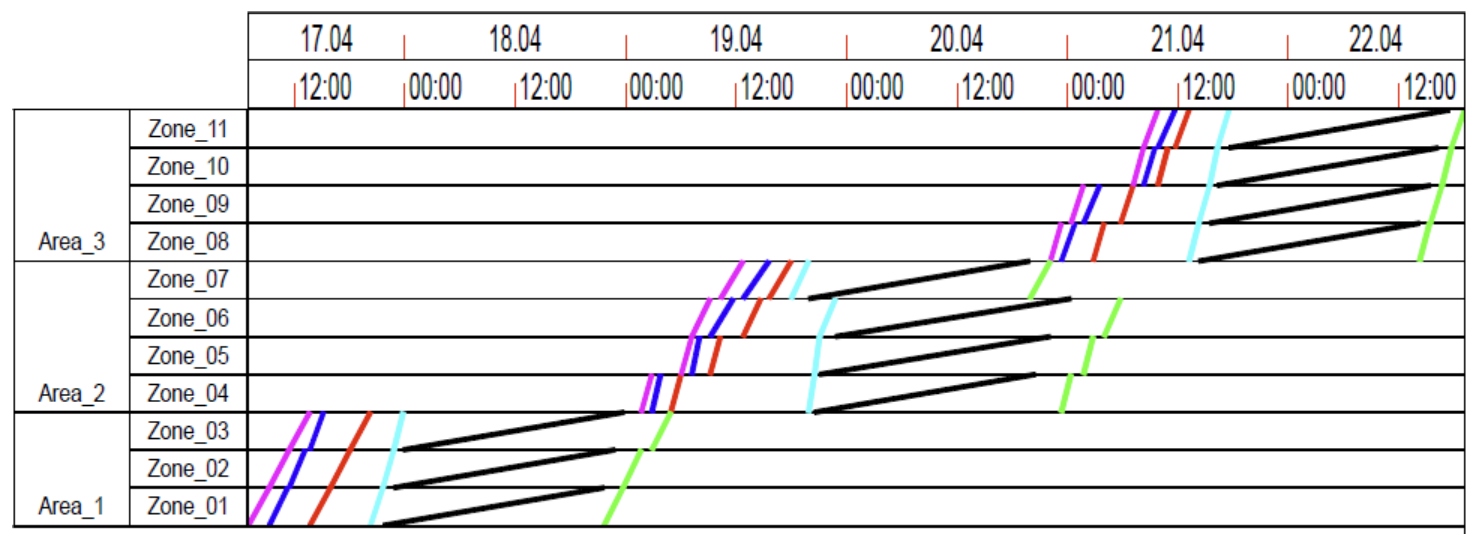

Figure 7: Line-of-balance chart - CP-B:

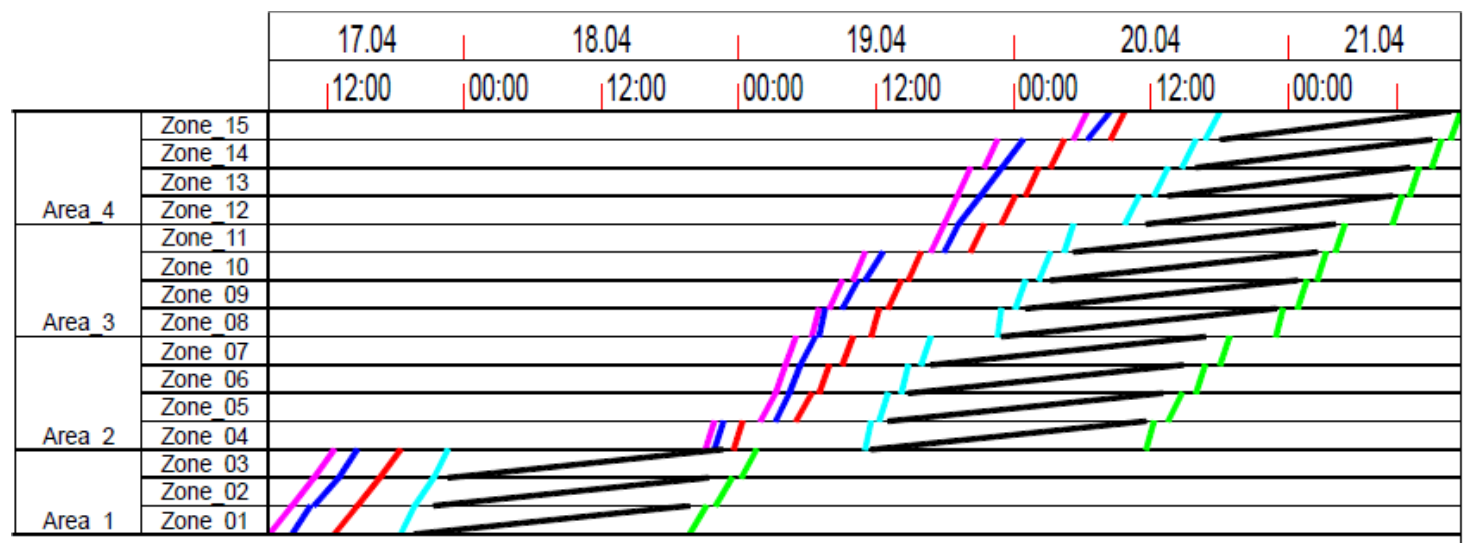

Figure 8: Line-of-balance chart - CP-D

$\rightleftarrows$ Setting1 $\rightleftarrows$ Reinforcing $\rightleftarrows$ Setting2 $\rightleftarrows$ Pouring $\rightleftarrows$ Striking 


\section{CONCLUSION}

Practical solutions for scheduling the execution of cast in-situ concrete constructions show breaks in location flow. This interrupts the on-site work and reduces the general production flow. We identified one reason as being spatial conflicts due to topological dependencies between casting segments in the predecessor and successor pour cycle. With the introduction of a new planning design by separating casting segments through nodes with high topological dependencies (NWHTDs), we can create solutions with fewer dependencies. Our simulation results show that such an optimised solution can achieve a better production flow. The simulation does not consider the material flow. More inventory (formwork panels) might arise from using our proposed planning design, which could be a disadvantage. We think that production flow could be improved by using a combination of precast elements to construct the NWHTDs, as well as cast in-situ concrete for the casting segments between them. This needs to be analysed in further research.

\section{ACKNOWLEDGEMENTS}

The authors would like to thank the companies Pöttinger GmbH \& Co. KG, Doka GmbH, Peri GmbH and Dr. Prautsch \& Partner Ingenieure PPI - Informatik for providing information to assist in the development and validation of our work. This research has been supported by the Bavarian Research Foundation.

\section{REFERENCES}

Ballard, G., \& Howell, G. (1998, August). What kind of production is construction. In Proc. 6 th Annual Conf. Int'l. Group for Lean Construction (pp. 13-15).

Ballard, G., \& Howell, G. (2003). Lean project management. Building Research \& Information, 31(2), 119-133.

Ballard, G., Koskela, L., Howell, G., \& Zabelle, T. (2001, August). Production system design in construction. In Annual Conference of the International Group for Lean Construction (Vol. 9).

Biruk, S., and Jaskowski, P. (2017). "Optimization of Vertical Formwork Layout Plans Using Mixed Integer Linear Programming." Int J Civ Eng, 10.1007/s40999-016-00906, 125-133.

Borrmann, A., \& Rank, E. (2009). Topological analysis of 3D building models using a spatial query language. Advanced Engineering Informatics, 23(4), 370-385.Cormen, T. H. (2005). Introduction to algorithms, MIT Press; McGraw-Hill, Cambridge, Mass., Boston, Mass.

Frandson, A., Berghede, K., \& Tommelein, I. D. (2013, August). Takt time planning for construction of exterior cladding. In Proc. 21st Ann. Conf. of the Int'l Group for Lean Construction.

Frandson, A. G., Seppänen, O., \& Tommelein, I. D. (2015, July). Comparison between location based management and takt time planning. In Proceedings of the 23rd Annual Conference of the International Group for Lean Construction (pp. 28-31). Perth: IGLC. 
Howell, G. A. (1999, July). What is lean construction-1999. In Proceedings IGLC (Vol. 7, p. 1)..

Häringer, P., and Borrmann, A. (2018). "Evaluation of a Case Study to Design a BIMBased Cycle Planning Concept." 26th Annual Conference of the International Group for Lean Construction, 10.24928/2018/0224.

Hofstadler, C. (2007). Wahrscheinlichkeitsüberlegungen-Berechnung der Dauer. Bauablaufplanung und Logistik im Baubetrieb, 413-429.

Kenley, R., \& Seppänen, O. (2006). Location-based management for construction: Planning, scheduling and control. Routledge.

Sacks, R. (2016). What constitutes good production flow in construction?. Construction management and economics, 34(9), 641-656.

Seppänen, O., Ballard, G., \& Pesonen, S. (2010). The Combination of Last Planner System and Location-Based Management System. Lean construction journal. 
Häringer, P. and Borrmann A

Proceedings IGLC - 27, July 2019, Dublin, Ireland 